\title{
Oscillator strengths for lines of astrophysical interest in Rh II
}

\author{
P. Quinet ${ }^{1,2}$, E. Biémont ${ }^{1,2}$, P. Palmeri ${ }^{1}$, L. Engström ${ }^{3}$, H. Hartman ${ }^{4}$, H. Lundberg ${ }^{3}$, and H. Nilsson ${ }^{4}$ \\ ${ }^{1}$ Astrophysique et Spectroscopie, Université de Mons - UMONS, 7000 Mons, Belgium \\ e-mail: quinet@umons.ac. be \\ 2 IPNAS, Université de Liège, Sart Tilman, 4000 Liège, Belgium \\ 3 Department of Physics, Lund University, PO Box 118, 22100 Lund, Sweden \\ ${ }^{4}$ Lund Observatory, Lund University, PO Box 43, 22100 Lund, Sweden
}

Received 4 October 2011 / Accepted 24 October 2011

\section{ABSTRACT}

\begin{abstract}
Aims. This work reports oscillator strengths for transitions of astrophysical interest in singly ionized rhodium.
Methods. Seventeen radiative lifetimes in $\mathrm{Rh}^{+}$have been measured with the time-resolved laser-induced fluorescence technique and combined with theoretical branching fractions calculated using a relativistic Hartree-Fock model including core-polarization effects to obtain oscillator strengths.

Results. On the basis of the good agreement between theory and experiment for the lifetimes, new reliable oscillator strengths have been deduced for a set of $113 \mathrm{Rh}$ II transitions in the spectral range 153-418 nm.
\end{abstract}

Key words. atomic processes - atomic data

\section{Introduction}

The investigation of high resolution astronomical spectra depends directly on reliable atomic data such as transition probabilities and oscillator strengths. Although many data are now available from modern experimental techniques or sophisticated theoretical methods, they are still insufficient to meet all the needs of astrophysicists. This is particularly true for singly ionized rhodium for which no radiative data have been published so far in the literature despite the fact that several Rh II lines have been identified in different astrophysical spectra such as the solar spectrum (Moore et al. 1966) and the spectra of the HgMn type star $\chi$ Lupi (Lundberg et al. 1998), the super-rich mercury star HD 65949, the HgMn star HD 175640 and the peculiar Przybylski's star HD 101065 (Cowley 2009). As examples, for the three latter stars, lines observed at $\lambda=309.35$, $316.22,318.79,320.73,324.05,330.74$ and $347.78 \mathrm{~nm}$ were identified as radiative transitions in Rh II.

Rhodium has one stable isotope, ${ }^{103} \mathrm{Rh}$, and 19 short-lived isotopes and isomers. In stellar nucleosynthesis, it is produced by both the $r$ - and $s$-processes. The spectrum of singly ionized rhodium belongs to the $\mathrm{Ru} \mathrm{I}$ isoelectronic sequence with $4 d^{8}{ }^{3} \mathrm{~F}_{4}$ as the ground state. The laboratory term analysis was performed by Sancho (1958) who classified 814 spectral lines extending from 118.7 to $328.8 \mathrm{~nm}$ and established a list of 36 even and 84 odd energy levels belonging to the $4 d^{8}, 4 d^{7} 5 \mathrm{~s}$ and $4 d^{7} 5 p$ configurations. This work was taken as reference in subsequent atomic data compilations by Moore (1971), Meggers et al. (1975) and Reader et al. (1980). To our knowledge, the only determination of experimental transition probabilities in Rh II is due to Corliss \& Bozman (1962) but their arc measurements have been recognized to be affected by large systematic errors.

* Table 3 is also available at the CDS via anonymous ftp to cdsarc.u-strasbg. fr (130.79.128.5) or via

http://cdsarc.u-strasbg.fr/viz-bin/qcat?]/A+A/537/A74
In the present paper, we report on time-resolved laserinduced fluorescence (TR-LIF) lifetime measurements for 17 levels in Rh II. These new experimental lifetimes have been used to assess the reliability of theoretical results obtained within the framework of the relativistic Hartree-Fock (HFR) approach (Cowan 1981) modified by the inclusion of core-polarization effects (HFR+CPOL) (Quinet et al. 1999). From the combination of experimental lifetimes and theoretical branching fractions, it has been possible to obtain a new set of semi-empirical oscillator strengths for selected transitions of astrophysical interest. This work is an extension to longer wavelengths of our recent paper focused on VUV lines in singly ionized rhodium (Quinet et al. 2011).

\section{Radiative lifetime measurements}

The experimental setup used in the present experiments has been described elsewhere (Bergström et al. 1988; Xu et al. 2004; Nilsson et al. 2010) and only a brief description will be given here. The lifetime measurements were performed on ions in a laser-generated plasma employing the time-resolved laserinduced-fluorescence technique. Nd-YAG laser pulses at $532 \mathrm{~nm}$ with a duration of $10 \mathrm{~ns}$ were focused onto the surface of a rotating rhodium target in a vacuum chamber with a background pressure of about $10^{-5}$ mbar. For the excitation of the investigated levels, the expanding rhodium plasma was crossed at right angle by a pulsed laser beam tuned to a resonant transition of the upper level of interest. The laser used a DCM dye and was pumped by a second Nd:YAG laser. The pump laser pulses had a duration of $10 \mathrm{~ns}$ and were compressed to about $1.5 \mathrm{~ns}$ utilizing stimultated Brillouin scattering in a water cell. The two Nd:YAG lasers were synchronized by a pulse generator and had a repetition rate of $10 \mathrm{~Hz}$. For the selective excitation of the investigated $\mathrm{Rh}^{+}$ levels the corresponding wavelengths were achieved using the third harmonic of the red dye laser light, obtained by harmonic 


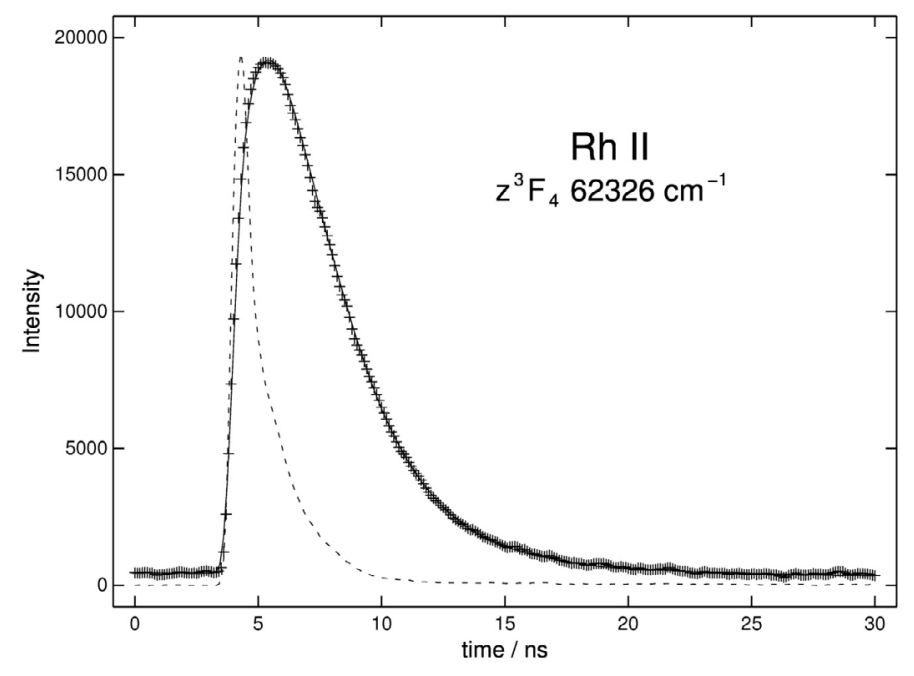

Fig. 1. Decay of the $4 d^{7}\left({ }^{4} F\right) 5 p z^{3} F_{4}^{\circ}$ level at $62326 \mathrm{~cm}^{-1}$, with an evaluated lifetime of $2.4 \pm 0.2 \mathrm{~ns}$. Background subtracted data points with typical error bars are plotted together with a fitted single exponential decay convoluted by the measured laser pulse (solid line). The actual measurement covers more than $90 \mathrm{~ns}$. The dashed curve shows the recorded laser pulse with a $F W H M$ of $1.1 \mathrm{~ns}$.

generation in KDP and BBO crystals and, if necessary, shifted by stimulated Raman scattering in hydrogen gas. In the measurements metastable levels of the same parity as the ground level were used as starting point for the selective excitation. These levels, with energies between 16885 and $20647 \mathrm{~cm}^{-1}$, are only populated in an early stage of the plasma expansion. The measurements were performed about $5 \mathrm{~mm}$ above the target and the delay between the plasma-generating and excitation pulses was around $300 \mathrm{~ns}$. This corresponds to an ion velocity larger than $10^{4} \mathrm{~m} / \mathrm{s}$ in the expanding plasma.

The TR-LIF signal was selected by a $1 / 8 \mathrm{~m}$ monochromator, detected by a fast photomultiplier tube with rise time $0.15 \mathrm{~ns}$ and digitized by an oscilloscope with $2.5 \mathrm{GHz}$ analogue bandwidth. The temporal shape of the exciting laser pulses was recorded simultaneously using a photo diode. Each pair of curves were formed by averaging over 1000 laser pulses and then transferred to a PC for further treatment. The lifetimes were obtained after fitting the LIF curve with an exponential convoluted with the recorded laser pulse. An example of an experimental recording is shown in Fig. 1. In Table 1 the lifetime values are given together with the lower level used for the selective excitation, excitation wavelength, non-linear processes to obtain this wavelength from a red dye laser and strongest detection channel. Whenever possible recordings were made on several detection wavelengths. The lifetime values are averages of more than 10 recordings performed at at least two different occasions. The uncertainties in the table are mainly due to variation in results between repeated measurements and possible systematic errors, which were carefully checked for (Sikström et al. 2002). They correspond to at least a $95 \%$ confidence interval of the various measurements. Due to recombination in the plasma LIF curves for all investigated states were more or less affected by background light. The problem could not be avoided since the metastable platform levels were only populated during the early stage of the plasma evolution. The background was subtracted in the fitting procedure but remains as a major contribution to the error bars in the present measurements.

\section{Transition probability and oscillator strength calculations}

As the $\mathrm{Rh}^{+}$ion belongs to the ruthenium isoelectronic sequence, we considered a relativistic Hartree-Fock model with core-polarization (HFR+CPOL) similar to the one used with success in Ru I (Fivet et al. 2009). More precisely, the configurations included in the configuration interaction expansions were the following: $4 d^{8}+4 d^{7} 5 s+4 d^{7} 6 s+4 d^{7} 5 d+4 d^{7} 6 d+4 d^{6} 5 s^{2}$ $+4 d^{6} 5 p^{2}+4 d^{6} 5 d^{2}+4 d^{6} 5 s 6 s+4 d^{6} 5 s 5 d+4 d^{6} 5 s 6 d$ (even parity) and $4 d^{7} 5 p+4 d^{7} 6 p+4 d^{7} 4 f+4 d^{7} 5 f+4 d^{6} 5 s 5 p+4 d^{6} 5 s 6 p+$ $4 d^{6} 5 p 5 d+4 d^{6} 5 p 6 s$ (odd parity). The ionic core considered for the core-polarization model potential and the correction to the dipole operator was a Mo-like core, i.e. a $4 \mathrm{~d}^{6} \mathrm{Rh}$ IV core. The dipole polarizability, $\alpha_{\mathrm{d}}$, for such a core is, according to Fraga et al. (1976), equal to $4.79 \mathrm{a}_{0}^{3}$. For the cut-off radius, $r_{\mathrm{c}}$, we used the HFR mean value $\langle r\rangle$ of the outermost $4 \mathrm{~d}$ core orbital, i.e. $1.52 a_{0}$.

Some radial integrals, considered as free parameters, were then adjusted with a least-squares optimization program minimizing the discrepancies between the calculated Hamiltonian eigenvalues and the experimental energy levels from Sancho (1958). More precisely, for the $4 d^{8}, 4 d^{7} 5 \mathrm{~s}$ and $4 d^{7} 5 \mathrm{p}$ configurations, the average energies $\left(E_{\mathrm{av}}\right)$, the electrostatic direct $\left(F^{k}\right)$ and exchange $\left(G^{k}\right)$ integrals, the spin-orbit $\left(\zeta_{n l}\right)$ and the effective interaction $(\alpha)$ parameters were allowed to vary during the fitting process. An additional effective operator $(\beta)$ for the $4 d^{7} 5 \mathrm{~s}$ configuration was also included in the adjustment. All other Slater integrals were scaled down by a factor 0.85 following a well-established procedure (Cowan 1981). The designations of the even levels at 8164.4 and $11643.7 \mathrm{~cm}^{-1}$ in Sancho (1958) were interchanged as indicated by the calculations of Shadmi (1961). The even level at $35012.0 \mathrm{~cm}^{-1}$ was excluded from the fit because its designation as $4 d^{7}\left({ }^{2} \mathrm{P}\right) 5 \mathrm{~s} \mathrm{~b} \mathrm{~b}^{3} \mathrm{P}_{0}$ by Sancho (1958) appeared questionable. In fact the predicted eigenvalue for this state was found to be more than $1000 \mathrm{~cm}^{-1}$ above the experimental value. Actually, the closest even eigenstate with $J=0$ is $4 \mathrm{~d}^{8}$ ${ }^{1} \mathrm{~S}_{0}$. Unfortunately, a comparison between the predicted transition probabilities and the intensities observed by Sancho (1958) for the 6 classified lines (of which 2 are doubly classified) involving this level was not conclusive in asserting this alternative designation. The standard deviations of the fits were $50 \mathrm{~cm}^{-1}$ for the even parity (35 levels, 12 parameters) and $111 \mathrm{~cm}^{-1}$ for the odd parity ( 84 levels, 9 parameters). The adopted radial parameter values with their standard deviations are given in Table 2.

Theoretical radiative lifetimes obtained in the present study are compared with the experimental values in Table 1. As shown in this table, the agreement between theory and experiment is good. However, the HFR+CPOL lifetimes are systematically about $10 \%$ longer than the measurements. This is probably due to the fact that core-polarization effects were slightly overestimated in our physical model. It is worth mentioning that calculated lifetimes were found to decrease by up to $5 \%$ when using a dipole polarizability equal to $4.3 a_{0}^{3}$, i.e. $10 \%$ smaller than the one published by Fraga et al. (1976) for Rh IV. It was also verified that a variation of $10 \%$ of the cut-off radius did not modify the computed lifetimes.

Considering the experimental lifetime values and the theoretical branching fractions as obtained in the present work, it is possible to derive normalized transition probabilities and oscillator strengths. In Table 3 , we present the $g A$ - and $\log g f$-values for the strongest lines $(\log g f>-2.0)$ depopulating the levels for which the experimental lifetimes are reported in Table 1. 
P. Quinet et al.: Oscillator strengths for lines of astrophysical interest in Rh II

Table 1. Experimental and calculated lifetimes obtained in the present work for selected levels within the $4 \mathrm{~d}^{7}\left({ }^{4} \mathrm{~F}\right) 5 \mathrm{p}$ configuration of Rh II.

\begin{tabular}{|c|c|c|c|c|c|c|c|}
\hline Level & Energy $\left(\mathrm{cm}^{-1}\right)^{a}$ & Origin $\left(\mathrm{cm}^{-1}\right)^{a}$ & $\begin{array}{c}\text { Excitation } \\
\lambda_{\text {vac }}(\mathrm{nm})\end{array}$ & Conversion $^{b}$ & Detection (nm) & $\tau_{\exp }(\mathrm{ns})$ & $\tau_{\text {calc }}(\mathrm{ns})$ \\
\hline $\mathrm{z}^{5} \mathrm{~F}_{4}^{\circ}$ & 56547.3 & 18540.4 & 263.03 & $3 \omega+2 S$ & 252 & $3.9 \pm 0.5$ & 4.4 \\
\hline $\mathrm{z}^{5} \mathrm{~F}_{5}^{\circ}$ & 57020.8 & 18540.4 & 259.80 & $3 \omega+2 S$ & 249 & $3.8 \pm 0.3$ & 4.0 \\
\hline $\mathrm{z}^{5} \mathrm{~F}_{3}^{\circ}$ & 58358.5 & 19792.4 & 259.22 & $3 \omega+2 S$ & 251 & $3.8 \pm 0.3$ & 4.2 \\
\hline $\mathrm{z}^{5} \mathrm{~F}_{2}^{\mathrm{o}}$ & 59698.6 & 20646.9 & 255.99 & $3 \omega+2 S$ & 251 & $3.8 \pm 0.3$ & 4.2 \\
\hline $\mathrm{z}^{5} \mathrm{D}_{4}^{\circ}$ & 59161.5 & 16884.8 & 236.47 & $3 \omega+\mathrm{S}$ & $246 / 304$ & $3.3 \pm 0.2$ & 3.9 \\
\hline $\mathrm{z}^{5} \mathrm{D}_{3}^{\circ}$ & 60448.4 & 18540.4 & 238.55 & $3 \omega+S$ & $246 / 303$ & $3.4 \pm 0.4$ & 3.9 \\
\hline $\mathrm{z}^{5} \mathrm{D}_{2}^{\mathrm{o}}$ & 61355.9 & 19792.4 & 240.52 & $3 \omega+S$ & $246 / 302$ & $3.7 \pm 0.5$ & 4.0 \\
\hline $\mathrm{z}^{5} \mathrm{G}_{6}^{\circ}$ & 59702.4 & 16884.8 & 233.48 & $3 \omega+\mathrm{S}$ & 233 & $3.0 \pm 0.2$ & 3.1 \\
\hline $\mathrm{z}^{5} \mathrm{G}_{5}^{\circ}$ & 59729.4 & 16884.8 & 233.33 & $3 \omega+S$ & 243 & $3.5 \pm 0.2$ & 4.0 \\
\hline $\mathrm{z}^{5} \mathrm{G}_{4}^{\circ}$ & 61173.1 & 16884.8 & 225.72 & $3 \omega+S$ & 242 & $3.3 \pm 0.2$ & 3.5 \\
\hline $\mathrm{z}^{5} \mathrm{G}_{3}^{\mathrm{o}}$ & 61939.8 & 18540.4 & 230.35 & $3 \omega+S$ & 242 & $3.3 \pm 0.3$ & 3.6 \\
\hline $\mathrm{z}^{5} \mathrm{G}_{2}^{\mathrm{o}}$ & 62288.3 & 20646.9 & 240.07 & $3 \omega+S$ & $243 / 235$ & $3.3 \pm 0.3$ & 3.6 \\
\hline $\mathrm{z}^{3} \mathrm{G}_{5}^{\circ}$ & 62194.4 & 16884.8 & 220.64 & $3 \omega$ & 272 & $3.2 \pm 0.2$ & 3.5 \\
\hline $\mathrm{z}^{3} \mathrm{G}_{4}^{\circ}$ & 63959.5 & 18540.4 & 220.10 & $3 \omega$ & 274 & $3.4 \pm 0.2$ & 3.7 \\
\hline$z^{3} G_{3}^{\circ}$ & 65321.2 & 18540.4 & 213.69 & $3 \omega$ & 274 & $2.0 \pm 0.2$ & 2.4 \\
\hline $\mathrm{z}^{3} \mathrm{~F}_{4}^{\circ}$ & 62326.1 & 16884.8 & 219.99 & $3 \omega$ & 271 & $2.4 \pm 0.2$ & 2.8 \\
\hline $\mathrm{z}^{3} \mathrm{~F}_{3}^{\mathrm{o}}$ & 63454.9 & 18540.4 & 222.58 & $3 \omega$ & 278 & $2.3 \pm 0.3$ & 2.5 \\
\hline
\end{tabular}

Notes. ${ }^{(a)}$ From Sancho (1958); ${ }^{(b)} 3 \omega$ : frequency tripling, $S$ : Stokes Raman shifting.

Table 2. Adopted radial parameters for the $4 d^{8}, 4 d^{7} 5 s$ and $4 d^{7} 5 p$ configurations.

\begin{tabular}{|c|c|c|c|c|}
\hline Configuration & Parameter & $\begin{array}{c}\text { Ab initio } \\
\left(\mathrm{cm}^{-1}\right)\end{array}$ & $\begin{array}{c}\text { Fitted } \\
\left(\mathrm{cm}^{-1}\right)\end{array}$ & Ratio \\
\hline \multirow[t]{5}{*}{$4 d^{8}$} & $E_{\mathrm{av}}$ & 10882 & $10421 \pm 21$ & \\
\hline & $F^{2}(4 \mathrm{~d}, 4 \mathrm{~d})$ & 65044 & $51938 \pm 222$ & 0.80 \\
\hline & $F^{4}(4 \mathrm{~d}, 4 \mathrm{~d})$ & 42149 & $35804 \pm 554$ & 0.85 \\
\hline & $\alpha$ & 0 & $21 \pm 5$ & \\
\hline & $\zeta_{4 \mathrm{~d}}$ & 1163 & $1180 \pm 20$ & 1.01 \\
\hline \multirow[t]{7}{*}{$4 d^{7} 5 s$} & $E_{\mathrm{av}}$ & 36719 & $37656 \pm 26$ & \\
\hline & $F^{2}(4 d, 4 d)$ & 68421 & $54908 \pm 332$ & 0.80 \\
\hline & $F^{4}(4 \mathrm{~d}, 4 \mathrm{~d})$ & 44587 & $39300 \pm 466$ & 0.88 \\
\hline & $\alpha$ & 0 & $32 \pm 2$ & \\
\hline & $\beta$ & 0 & $-462 \pm 142$ & \\
\hline & $\zeta_{4 d}$ & 1256 & $1260 \pm 10$ & 1.00 \\
\hline & $G^{2}(4 \mathrm{~d}, 5 \mathrm{~s})$ & 13647 & $11376 \pm 42$ & 0.83 \\
\hline \multirow[t]{9}{*}{$4 d^{7} 5 p$} & $E_{\mathrm{av}}$ & 74500 & $77980 \pm 16$ & \\
\hline & $F^{2}(4 \mathrm{~d}, 4 \mathrm{~d})$ & 69029 & $55106 \pm 135$ & 0.80 \\
\hline & $F^{4}(4 \mathrm{~d}, 4 \mathrm{~d})$ & 45033 & $38083 \pm 194$ & 0.85 \\
\hline & $\alpha$ & 0 & $24 \pm 2$ & \\
\hline & $\zeta_{4 \mathrm{~d}}$ & 1269 & $1269 \pm 15$ & 1.00 \\
\hline & $\zeta_{5 p}$ & 1135 & $1406 \pm 33$ & 1.24 \\
\hline & $F^{2}(4 \mathrm{~d}, 5 \mathrm{p})$ & 17482 & $14722 \pm 134$ & 0.84 \\
\hline & $G^{1}(4 \mathrm{~d}, 5 \mathrm{p})$ & 7027 & $5339 \pm 57$ & 0.76 \\
\hline & $G^{3}(4 \mathrm{~d}, 5 \mathrm{p})$ & 5685 & $3798 \pm 144$ & 0.67 \\
\hline
\end{tabular}

\section{Conclusions}

A new set of transition probabilities and oscillator strengths has been obtained for transitions depopulating 17 levels of Rh II belonging to the $4 d^{7}\left({ }^{4} F\right) 5 p$ configuration. These results have been obtained from a combination of experimental lifetimes measured by laser-induced fluorescence spectroscopy and HFR+CPOL branching fractions.
Acknowledgements. This work was financially supported by the Integrated Initiative of Infrastructure Project LASERLAB-EUROPE, contract RII3-CT2003-506350, the Swedish Research Council through the Linnaeus grant and grant 2006-3085, the Knut and Alice Wallenberg Foundation and the Belgian FRS-FNRS. E.B., P.Q. and P.P. are, respectively, Research Director, Senior Research Associate and Research Associate of the FRS-FNRS. They are grateful to the Swedish team for the warm hospitality enjoyed at the Lund Laser Centre during the measurements.

\section{References}

Bergström, H., Faris, H., Hallstadius, G. W., et al. 1988, Z. Phys. D, 8, 17 Biémont, E., Palmeri, P., Quinet, P., et al. 2001, MNRAS, 328, 1085

Corliss, C. H., \& Bozman, W. R. 1962, in Nat. Bur. Stand. Monogr. (Washington DC: US Department of Commerce), 53

Cowan, R. D. 1981, The Theory of Atomic Structure and Spectra (Berkeley: California University Press)

Cowley, C. R. 2009, http://www . astro.1sa.umich.edu /cowley

Fivet, V., Quinet, P., Palmeri, P., et al. 2009, MNRAS, 396, 2124

Fraga, S., Karwowski, J., \& Saxena, K. M. S. 1976, Handbook of Atomic Data (Amsterdam: Elsevier)

Lundberg, H., Johansson, S., Litzén, U., Wahlgren, G. M., \& Leckrone, S. 1998, in The Scientific Impact of the Goddard High Resolution Spectrograph, ASP Conf. Ser., 143, 343

Meggers, W. F., Corliss, C. H., \& Scribner, B. F. 1975, Nat. Bur. Stand. NBS, Monograph (Washington DC: US Department of Commerce), 145

Moore, C. E. 1971, Nat. Stand. Ref. Data Ser. NSRDS-NBS, 35, III

Moore, C. E., Minnaert, M. G. J., \& Houtgast, J. 1966, The Solar Spectrum $2935 \AA$ to $8770 \AA$ Å, NBS Monograph 61 (Washington DC: US Department of Commerce)

Nilsson, H., Hartman, H., Engström, L., et al. 2010, A\&A, 511, A16

Quinet, P., Palmeri, P., Biémont, E., et al. 1999, MNRAS, 307, 934

Quinet, P., Biémont, E., Palmeri, P., et al. 2011, J. Elec. Spectr. Rel. Phen., 184, 174

Reader, J., Corliss, C. H., Wiese, W. L., \& Martin, G. A. 1980, Nat. Stand. Ref. Data Ser. NSRDS-NBS, 68

Sancho, F. J. 1958, Anales Real. Soc. Esp. Fis. Quim., 54A, 41

Shadmi, Y. 1961, Bull. Res. Council Israel, 9F, 141

Sikström, C. M., Nilsson, H., Litzén, U., Blom, A., \& Lundberg, H. 2002, J. Quant. Spec. Radiat. Transf., 74, 355

Xu, H. L., Persson, A., Svanberg, S., et al. 2004, Phys. Rev. A, 70, 042508

Zhang, Z. G., Li, Z. S., Svanberg, S., et al. 2001, Eur. Phys. J. D, 15, 301 
Table 3. Transition probabilities and oscillator strengths for Rh II selected lines. $X(Y)$ stands for $X \times 10^{Y}$. Only transitions with log $g f \geq-2.0$ are listed in the table.

\begin{tabular}{|c|c|c|c|c|c|c|c|c|c|}
\hline \multirow[t]{2}{*}{$\lambda^{a}(\mathrm{~nm})$} & \multirow[t]{2}{*}{ Int. ${ }^{a}$} & \multicolumn{2}{|c|}{ Lower level $^{a}$} & \multicolumn{2}{|c|}{ Upper level $^{a}$} & \multicolumn{2}{|c|}{$\mathrm{HFR}+\mathrm{CPOL}^{b}$} & \multicolumn{2}{|c|}{ Normalized $^{c}$} \\
\hline & & $E\left(\mathrm{~cm}^{-1}\right)$ & Desig. & $E\left(\mathrm{~cm}^{-1}\right)$ & Desig. & $g A\left(\mathrm{~s}^{-1}\right)$ & $\log g f$ & $g A\left(\mathrm{~s}^{-1}\right)$ & $\log g f$ \\
\hline 153.091 & 100 & 0.0 & $a^{3} F_{4}$ & 65321.2 & $\mathrm{z}^{3} \mathrm{G}_{3}^{\circ}$ & $8.55(8)$ & -0.52 & 1.03(9) & -0.44 \\
\hline 156.348 & 100 & 0.0 & $a^{3} F_{4}$ & 63959.5 & $\mathrm{z}^{3} \mathrm{G}_{4}^{\circ}$ & 1.71(8) & -1.20 & $1.86(8)$ & -1.17 \\
\hline 157.591 & 100 & 0.0 & $a^{3} F_{4}$ & 63454.9 & $\mathrm{z}^{3} \mathrm{~F}_{3}^{\circ}$ & $2.17(8)$ & -1.09 & $2.36(8)$ & -1.06 \\
\hline 158.932 & 90 & 2401.3 & $a^{3} F_{3}$ & 65321.2 & $\mathrm{z}^{3} \mathrm{G}_{3}^{\circ}$ & $1.92(8)$ & -1.14 & $2.30(8)$ & -1.06 \\
\hline 160.445 & 500 & 0.0 & $a^{3} F_{4}$ & 62326.1 & $\mathrm{z}^{3} \mathrm{~F}_{4}^{\circ}$ & $1.14(9)$ & -0.36 & $1.33(9)$ & -0.29 \\
\hline 160.786 & 100 & 0.0 & $a^{3} F_{4}$ & 62194.4 & $\mathrm{z}^{3} \mathrm{G}_{5}^{\circ}$ & $1.02(8)$ & -1.40 & $1.12(8)$ & -1.36 \\
\hline 162.447 & 200 & 2401.3 & $a^{3} F_{3}$ & 63959.5 & $\mathrm{z}^{3} \mathrm{G}_{4}^{\circ}$ & $6.06(7)$ & -1.62 & $6.59(7)$ & -1.58 \\
\hline 163.472 & 200 & 0.0 & $a^{3} F_{4}$ & 61173.1 & $\mathrm{z}^{5} \mathrm{G}_{4}^{\circ}$ & $2.76(8)$ & -0.96 & 2.93(8) & -0.93 \\
\hline 163.788 & 200 & 2401.3 & $a^{3} F_{3}$ & 63454.9 & $\mathrm{z}^{3} \mathrm{~F}_{3}^{\circ}$ & $6.72(8)$ & -0.57 & $7.30(8)$ & -0.53 \\
\hline 166.876 & 100 & 2401.3 & $a^{3} F_{3}$ & 62326.1 & $\mathrm{z}^{3} \mathrm{~F}_{4}^{\circ}$ & $1.66(8)$ & -1.16 & $1.94(8)$ & -1.09 \\
\hline 167.019 & 90 & 3580.7 & $a^{3} F_{2}$ & 63454.9 & $\mathrm{z}^{3} \mathrm{~F}_{3}^{\circ}$ & $1.39(8)$ & -1.24 & $1.51(8)$ & -1.20 \\
\hline 167.422 & 100 & 0.0 & $a^{3} F_{4}$ & 59729.4 & $\mathrm{z}^{5} \mathrm{G}_{5}^{\circ}$ & $1.41(8)$ & -1.23 & $1.61(8)$ & -1.17 \\
\hline 167.958 & 50 & 2401.3 & $a^{3} F_{3}$ & 61939.8 & $\mathrm{z}^{5} \mathrm{G}_{3}^{\circ}$ & $1.11(8)$ & -1.33 & $1.21(8)$ & -1.29 \\
\hline 170.337 & 50 & 3580.7 & $a^{3} F_{2}$ & 62288.3 & $\mathrm{z}^{5} \mathrm{G}_{2}^{\circ}$ & $4.60(7)$ & -1.70 & $5.02(7)$ & -1.66 \\
\hline 174.958 & 100 & 8164.4 & $a^{1} D_{2}$ & 65321.2 & $\mathrm{z}^{3} \mathrm{G}_{3}^{\circ}$ & $1.74(8)$ & -1.10 & $2.09(8)$ & -1.02 \\
\hline 176.842 & 100 & 0.0 & $a^{3} F_{4}$ & 56547.3 & $\mathrm{z}^{5} \mathrm{~F}_{4}^{\circ}$ & $2.47(7)$ & -1.93 & $2.79(7)$ & -1.88 \\
\hline 180.864 & 100 & 8164.4 & $a^{1} D_{2}$ & 63454.9 & $z^{3} \mathrm{~F}_{3}^{\circ}$ & 1.63(8) & -1.10 & $1.77(8)$ & -1.06 \\
\hline $186.298^{*}$ & & 11643.7 & $a^{3} P_{2}$ & 65321.2 & $\mathrm{z}^{3} \mathrm{G}_{3}^{\circ}$ & $8.76(7)$ & -1.34 & $1.05(8)$ & -1.26 \\
\hline $193.008^{*}$ & & 11643.7 & $a^{3} P_{2}$ & 63454.9 & $\mathrm{z}^{3} \mathrm{~F}_{3}^{\circ}$ & $4.45(7)$ & -1.60 & $4.84(7)$ & -1.57 \\
\hline $213.696^{*}$ & & 18540.4 & $a^{5} F_{4}$ & 65321.2 & $\mathrm{z}^{3} \mathrm{G}_{3}^{\circ}$ & $1.33(7)$ & -2.04 & $1.60(7)$ & -1.96 \\
\hline 219.996 & 200 & 16884.8 & $a^{5} F_{5}$ & 62326.1 & $\mathrm{z}^{3} \mathrm{~F}_{4}^{0}$ & $9.18(7)$ & -1.17 & $1.07(8)$ & -1.11 \\
\hline 220.102 & 100 & 18540.4 & $a^{5} F_{4}$ & 63959.5 & $\mathrm{z}^{3} \mathrm{G}_{4}^{\circ}$ & 7.78(7) & -1.25 & $8.47(7)$ & -1.21 \\
\hline 220.635 & 100 & 16884.8 & $a^{5} F_{5}$ & 62194.4 & $\mathrm{z}^{3} \mathrm{G}_{5}^{\mathrm{o}}$ & $9.13(7)$ & -1.18 & $9.99(7)$ & -1.14 \\
\hline 223.771 & 50 & 20646.9 & $a^{5} F_{2}$ & 65321.2 & $\mathrm{z}^{3} \mathrm{G}_{3}^{\circ}$ & $2.85(8)$ & -0.67 & $3.42(8)$ & -0.59 \\
\hline 225.724 & 80 & 16884.8 & $a^{5} F_{5}$ & 61173.1 & $\mathrm{z}^{5} \mathrm{G}_{4}^{\circ}$ & $3.35(7)$ & -1.59 & $3.55(7)$ & -1.57 \\
\hline 226.343 & 50 & 19792.4 & $a^{5} F_{3}$ & 63959.5 & $\mathrm{z}^{3} \mathrm{G}_{4}^{\circ}$ & 7.51(8) & -0.24 & $8.17(8)$ & -0.20 \\
\hline 228.316 & 20 & 18540.4 & $a^{5} F_{4}$ & 62326.1 & $\mathrm{z}^{3} \mathrm{~F}_{4}^{\circ}$ & $1.91(7)$ & -1.82 & $2.23(7)$ & -1.76 \\
\hline 228.957 & 20 & 19792.4 & $a^{5} F_{3}$ & 63454.9 & $\mathrm{z}^{3} \mathrm{~F}_{3}^{\circ}$ & $3.88(7)$ & -1.52 & $4.22(7)$ & -1.48 \\
\hline 229.004 & 300 & 18540.4 & $a^{5} F_{4}$ & 62194.4 & $\mathrm{z}^{3} \mathrm{G}_{5}^{\mathrm{o}}$ & 1.85(9) & 0.16 & 2.02(9) & 0.20 \\
\hline 230.347 & 20 & 18540.4 & $a^{5} F_{4}$ & 61939.8 & $\mathrm{z}^{5} \mathrm{G}_{3}^{\circ}$ & $2.17(7)$ & -1.76 & $2.37(7)$ & -1.73 \\
\hline 233.330 & 300 & 16884.8 & $a^{5} F_{5}$ & 59729.4 & $\mathrm{z}^{5} \mathrm{G}_{5}^{\mathrm{o}}$ & $1.79(8)$ & -0.83 & $2.05(8)$ & -0.78 \\
\hline 233.477 & 1000 & 16884.8 & $a^{5} F_{5}$ & 59702.4 & $\mathrm{z}^{5} \mathrm{G}_{6}^{\circ}$ & $4.18(9)$ & 0.53 & $4.32(9)$ & 0.55 \\
\hline 233.530 & 10 & 20646.9 & $a^{5} F_{2}$ & 63454.9 & $\mathrm{z}^{3} \mathrm{~F}_{3}^{\circ}$ & $1.14(8)$ & -1.03 & $1.24(8)$ & -0.99 \\
\hline 234.489 & 80 & 18540.4 & $a^{5} F_{4}$ & 61173.1 & $\mathrm{z}^{5} \mathrm{G}_{4}^{\circ}$ & $1.34(7)$ & -1.96 & $1.42(7)$ & -1.93 \\
\hline 235.035 & 100 & 19792.4 & $a^{5} F_{3}$ & 62326.1 & $z^{3} \mathrm{~F}_{4}^{\circ}$ & $1.44(8)$ & -0.92 & $1.68(8)$ & -0.86 \\
\hline 236.467 & 100 & 16884.8 & $a^{5} F_{5}$ & 59161.5 & $\mathrm{z}^{5} \mathrm{D}_{4}^{\circ}$ & 1.17(8) & -1.00 & $1.38(8)$ & -0.94 \\
\hline 238.545 & 200 & 18540.4 & $a^{5} F_{4}$ & 60448.4 & $\mathrm{z}^{5} \mathrm{D}_{3}^{\circ}$ & $8.21(7)$ & -1.15 & $9.42(7)$ & -1.10 \\
\hline 240.071 & 80 & 20646.9 & $a^{5} F_{2}$ & 62288.3 & $\mathrm{z}^{5} \mathrm{G}_{2}^{\circ}$ & 4.73(7) & -1.39 & $5.16(7)$ & -1.35 \\
\hline 240.522 & 150 & 19792.4 & $a^{5} F_{3}$ & 61355.9 & $\mathrm{z}^{5} \mathrm{D}_{2}^{\circ}$ & $1.09(8)$ & -1.02 & $1.18(8)$ & -0.99 \\
\hline 241.584 & 500 & 19792.4 & $a^{5} F_{3}$ & 61173.1 & $\mathrm{z}^{5} \mathrm{G}_{4}^{\circ}$ & $1.80(9)$ & 0.20 & 1.91(9) & 0.22 \\
\hline 242.100 & 500 & 20646.9 & $a^{5} F_{2}$ & 61939.8 & $\mathrm{z}^{5} \mathrm{G}_{3}^{\circ}$ & $1.62(9)$ & 0.15 & $1.77(9)$ & 0.19 \\
\hline 242.709 & 300 & 18540.4 & $a^{5} F_{4}$ & 59729.4 & $\mathrm{z}^{5} \mathrm{G}_{5}^{\circ}$ & 1.54(9) & 0.14 & $1.76(9)$ & 0.19 \\
\hline 243.185 & 300 & 21180.0 & $a^{5} F_{1}$ & 62288.3 & $\mathrm{z}^{5} \mathrm{G}_{2}^{\circ}$ & $1.20(9)$ & 0.02 & $1.31(9)$ & 0.06 \\
\hline 245.571 & 200 & 20646.9 & $a^{5} F_{2}$ & 61355.9 & $\mathrm{z}^{5} \mathrm{D}_{2}^{\circ}$ & $1.00(9)$ & -0.04 & $1.08(9)$ & -0.01 \\
\hline 245.890 & 300 & 19792.4 & $a^{5} F_{3}$ & 60448.4 & $\mathrm{z}^{5} \mathrm{D}_{3}^{\circ}$ & $1.57(9)$ & 0.16 & $1.80(9)$ & 0.21 \\
\hline 246.103 & 300 & 18540.4 & $a^{5} F_{4}$ & 59161.5 & $\mathrm{z}^{5} \mathrm{D}_{4}^{\circ}$ & $2.09(9)$ & 0.28 & $2.47(9)$ & 0.35 \\
\hline 248.829 & 50 & 21180.0 & $a^{5} F_{1}$ & 61355.9 & $\mathrm{z}^{5} \mathrm{D}_{2}^{\circ}$ & $1.84(7)$ & -1.77 & $1.99(7)$ & -1.73 \\
\hline 249.079 & 150 & 16884.8 & $a^{5} F_{5}$ & 57020.8 & $\mathrm{z}^{5} \mathrm{~F}_{5}^{2}$ & 2.60(9) & 0.39 & 2.67(9) & 0.39 \\
\hline 250.276 & 50 & 25376.9 & $b^{3} F_{4}$ & 65321.2 & $\mathrm{z}^{3} \mathrm{G}_{3}^{\circ}$ & 2.53(8) & -0.62 & $3.04(8)$ & -0.55 \\
\hline 250.512 & 100 & 19792.4 & $a^{5} F_{3}$ & 59698.6 & $\mathrm{z}^{5} \mathrm{~F}_{2}^{0}$ & $9.82(8)$ & -0.03 & $1.09(9)$ & 0.01 \\
\hline 251.065 & 100 & 18540.4 & $a^{5} F_{4}$ & 58358.5 & $\mathrm{z}^{5} \mathrm{~F}_{3}^{\circ}$ & 1.43(9) & 0.13 & $1.58(9)$ & 0.17 \\
\hline 252.052 & 100 & 16884.8 & $a^{5} F_{5}$ & 56547.3 & $\mathrm{z}^{5} \mathrm{~F}_{4}^{\circ}$ & 1.79(9) & 0.24 & $2.02(9)$ & 0.28 \\
\hline 255.992 & 100 & 20646.9 & $a^{5} F_{2}$ & 59698.6 & $\mathrm{z}^{5} \mathrm{~F}_{2}^{\circ}$ & $1.44(8)$ & -0.85 & $1.59(8)$ & -0.81 \\
\hline 259.216 & 100 & 19792.4 & $a^{5} F_{3}$ & 58358.5 & $\mathrm{z}^{5} \mathrm{~F}_{3}^{\circ}$ & $1.24(8)$ & -0.90 & $1.37(8)$ & -0.86 \\
\hline 259.540 & 60 & 21180.0 & $a^{5} F_{1}$ & 59698.6 & $\mathrm{z}^{5} \mathrm{~F}_{2}^{\mathrm{o}}$ & $2.33(7)$ & -1.63 & $2.58(7)$ & -1.58 \\
\hline 259.795 & 10 & 18540.4 & $a^{5} F_{4}$ & 57020.8 & $\mathrm{z}^{5} \mathrm{~F}_{5}^{\circ}$ & $1.04(7)$ & -1.98 & $1.07(7)$ & -1.97 \\
\hline 262.541 & 150 & 25376.9 & $\mathrm{~b}^{3} \mathrm{~F}_{4}$ & 63454.9 & $\mathrm{z}^{3} \mathrm{~F}_{3}^{\circ}$ & 9.81(8) & 0.01 & $1.07(9)$ & 0.04 \\
\hline 263.033 & 100 & 18540.4 & $a^{5} F_{4}$ & 56547.3 & $\mathrm{z}^{5} \mathrm{~F}_{4}^{\circ}$ & $1.38(8)$ & -0.84 & $1.56(8)$ & -0.79 \\
\hline $263.900 *$ & & 27439.4 & $b^{3} F_{3}$ & 65321.2 & $\mathrm{z}^{3} \mathrm{G}_{3}^{\circ}$ & $2.26(8)$ & -0.63 & $2.71(8)$ & -0.55 \\
\hline 265.093 & 30 & 20646.9 & $a^{5} F_{2}$ & 58358.5 & $\mathrm{z}^{5} \mathrm{~F}_{3}^{0}$ & $1.50(7)$ & -1.80 & $1.66(7)$ & -1.76 \\
\hline 266.448 & 50 & 27801.4 & $a^{5} P_{3}$ & 65321.2 & $\mathrm{z}^{3} \mathrm{G}_{3}^{\circ}$ & $6.44(7)$ & -1.16 & 7.73(7) & -1.08 \\
\hline 270.560 & 150 & 25376.9 & $\mathrm{~b}^{3} \mathrm{~F}_{4}$ & 62326.1 & $\mathrm{z}^{3} \mathrm{~F}_{4}^{\mathrm{o}}$ & 1.53(9) & 0.23 & $1.79(9)$ & 0.29 \\
\hline
\end{tabular}


P. Quinet et al.: Oscillator strengths for lines of astrophysical interest in Rh II

Table 3. continued.

\begin{tabular}{|c|c|c|c|c|c|c|c|c|c|}
\hline \multirow[t]{2}{*}{$\lambda^{a}(\mathrm{~nm})$} & \multirow[t]{2}{*}{ Int. $^{a}$} & \multicolumn{2}{|c|}{ Lower level $^{a}$} & \multicolumn{2}{|c|}{ Upper level $^{a}$} & \multicolumn{2}{|c|}{$\mathrm{HFR}+\mathrm{CPOL}^{b}$} & \multicolumn{2}{|c|}{ Normalized $^{c}$} \\
\hline & & $E\left(\mathrm{~cm}^{-1}\right)$ & Desig. & $E\left(\mathrm{~cm}^{-1}\right)$ & Desig. & $g A\left(\mathrm{~s}^{-1}\right)$ & $\log g f$ & $g A\left(\mathrm{~s}^{-1}\right)$ & $\log g f$ \\
\hline 271.527 & 150 & 25376.9 & $\mathrm{~b}^{3} \mathrm{~F}_{4}$ & 62194.4 & $\mathrm{z}^{3} \mathrm{G}_{5}^{\circ}$ & $1.11(9)$ & 0.09 & $1.21(9)$ & 0.13 \\
\hline 273.420 & 10 & 25376.9 & $\mathrm{~b}^{3} \mathrm{~F}_{4}$ & 61939.8 & $\mathrm{z}^{5} \mathrm{G}_{3}^{\circ}$ & $1.41(7)$ & -1.80 & $1.54(7)$ & -1.76 \\
\hline 273.740 & 150 & 27439.4 & $b^{3} F_{3}$ & 63959.5 & $z^{3} G_{4}^{\circ}$ & 1.11(9) & 0.10 & $1.21(9)$ & 0.13 \\
\hline 273.992 & 150 & 28834.6 & $b^{3} F_{2}$ & 65321.2 & $z^{3} G_{3}^{\circ}$ & $6.61(8)$ & -0.13 & 7.93(8) & -0.05 \\
\hline 276.483 & 100 & 27801.4 & $a^{5} P_{3}$ & 63959.5 & $z^{3} G_{4}^{\circ}$ & $2.09(8)$ & -0.62 & $2.27(8)$ & -0.58 \\
\hline 277.577 & 100 & 27439.4 & $b^{3} F_{3}$ & 63454.9 & $\mathrm{z}^{3} \mathrm{~F}_{3}^{\circ}$ & $2.67(8)$ & -0.51 & $2.90(8)$ & -0.47 \\
\hline 279.278 & 100 & 25376.9 & $\mathrm{~b}^{3} \mathrm{~F}_{4}$ & 61173.1 & $\mathrm{z}^{5} \mathrm{G}_{4}^{\circ}$ & $9.25(7)$ & -0.97 & 9.81(7) & -0.94 \\
\hline 280.395 & 80 & 27801.4 & $a^{5} P_{3}$ & 63454.9 & $\mathrm{z}^{3} \mathrm{~F}_{3}^{\circ}$ & $5.93(7)$ & -1.16 & $6.45(7)$ & -1.12 \\
\hline 285.049 & 50 & 25376.9 & $\mathrm{~b}^{3} \mathrm{~F}_{4}$ & 60448.4 & $\mathrm{z}^{5} \mathrm{D}_{3}^{\circ}$ & $2.00(7)$ & -1.61 & $2.29(7)$ & -1.55 \\
\hline 286.560 & 30 & 27439.4 & $b^{3} F_{3}$ & 62326.1 & $\mathrm{z}^{3} \mathrm{~F}_{4}^{\circ}$ & $2.45(7)$ & -1.52 & $2.86(7)$ & -1.45 \\
\hline 289.560 & 2 & 27801.4 & $a^{5} P_{3}$ & 62326.1 & $\mathrm{z}^{3} \mathrm{~F}_{4}^{\circ}$ & $1.38(7)$ & -1.76 & 1.61(7) & -1.69 \\
\hline 289.763 & 100 & 27439.4 & $b^{3} F_{3}$ & 61939.8 & $z^{5} G_{3}^{\circ}$ & $3.61(7)$ & -1.34 & $3.94(7)$ & -1.30 \\
\hline 291.015 & 200 & 25376.9 & $b^{3} F_{4}$ & 59729.4 & $\mathrm{z}^{5} \mathrm{G}_{5}^{\circ}$ & $8.48(8)$ & 0.03 & $9.69(8)$ & 0.09 \\
\hline 292.680 & 100 & 28131.4 & $a^{5} P_{2}$ & 62288.3 & $\mathrm{z}^{5} \mathrm{G}_{2}^{\circ}$ & $1.10(7)$ & -1.85 & $1.20(7)$ & -1.81 \\
\hline 292.838 & 40 & 27801.4 & $a^{5} P_{3}$ & 61939.8 & $\mathrm{z}^{5} \mathrm{G}_{3}^{\circ}$ & $2.67(7)$ & -1.47 & 2.91(7) & -1.43 \\
\hline 294.756 & 30 & 27439.4 & $b^{3} F_{3}$ & 61355.9 & $\mathrm{z}^{5} \mathrm{D}_{2}^{\circ}$ & $2.04(7)$ & -1.57 & $2.21(7)$ & -1.54 \\
\hline 296.354 & 200 & 27439.4 & $b^{3} F_{3}$ & 61173.1 & $\mathrm{z}^{5} \mathrm{G}_{4}^{\circ}$ & $2.92(8)$ & -0.41 & $3.10(8)$ & -0.39 \\
\hline 298.830 & 40 & 28834.6 & $b^{3} F_{2}$ & 62288.3 & $\mathrm{z}^{5} \mathrm{G}_{2}^{\circ}$ & $6.77(7)$ & -1.05 & 7.39(7) & -1.00 \\
\hline 299.563 & 15 & 27801.4 & $a^{5} P_{3}$ & 61173.1 & $\mathrm{z}^{5} \mathrm{G}_{4}^{\mathrm{o}}$ & $1.90(7)$ & -1.59 & $2.02(7)$ & -1.57 \\
\hline 300.898 & 80 & 28131.4 & $a^{5} P_{2}$ & 61355.9 & $\mathrm{z}^{5} \mathrm{D}_{2}^{\circ}$ & $6.09(7)$ & -1.08 & $6.58(7)$ & -1.05 \\
\hline 301.978 & 100 & 28834.6 & $b^{3} F_{2}$ & 61939.8 & $\mathrm{z}^{5} \mathrm{G}_{3}^{\mathrm{o}}$ & $1.10(8)$ & -0.82 & $1.20(8)$ & -0.79 \\
\hline 302.857 & 80 & 27439.4 & $b^{3} F_{3}$ & 60448.4 & $\mathrm{z}^{5} \mathrm{D}_{3}^{\circ}$ & $1.16(7)$ & -1.79 & $1.33(7)$ & -1.74 \\
\hline 305.565 & 80 & 32604.7 & $a^{3} G_{4}$ & 65321.2 & $\mathrm{z}^{3} \mathrm{G}_{3}^{\mathrm{o}}$ & $4.56(7)$ & -1.20 & $5.47(7)$ & -1.12 \\
\hline 306.223 & 60 & 27801.4 & $a^{5} P_{3}$ & 60448.4 & $\mathrm{z}^{5} \mathrm{D}_{3}^{\circ}$ & $1.99(7)$ & -1.55 & $2.28(7)$ & -1.49 \\
\hline 309.344 & 100 & 28131.4 & $a^{5} P_{2}$ & 60448.4 & $\mathrm{z}^{5} \mathrm{D}_{3}^{\circ}$ & $4.96(7)$ & -1.15 & $5.69(7)$ & -1.09 \\
\hline 309.675 & 80 & 29073.0 & $a^{5} P_{1}$ & 61355.9 & $\mathrm{z}^{5} \mathrm{D}_{2}^{\circ}$ & $2.64(7)$ & -1.42 & $2.85(7)$ & -1.39 \\
\hline 310.191 & 15 & 31730.5 & $a^{3} G_{5}$ & 63959.5 & $\mathrm{z}^{3} \mathrm{G}_{4}^{\circ}$ & $1.73(7)$ & -1.60 & $1.88(7)$ & -1.57 \\
\hline 313.417 & 10 & 27801.4 & $a^{5} P_{3}$ & 59698.6 & $z^{5} \mathrm{~F}_{2}^{0}$ & $6.43(6)$ & -2.02 & 7.11(6) & -1.98 \\
\hline 315.144 & 40 & 27439.4 & $b^{3} F_{3}$ & 59161.5 & $\mathrm{z}^{5} \mathrm{D}_{4}^{\circ}$ & $9.82(6)$ & -1.83 & $1.16(7)$ & -1.76 \\
\hline 315.926 & 200 & 25376.9 & $b^{3} F_{4}$ & 57020.8 & $\mathrm{z}^{5} \mathrm{~F}_{5}^{\circ}$ & $1.06(8)$ & -0.80 & $1.09(8)$ & -0.79 \\
\hline 316.230 & 20 & 28834.6 & $b^{3} F_{2}$ & 60448.4 & $\mathrm{z}^{5} \mathrm{D}_{3}^{\circ}$ & $8.83(6)$ & -1.88 & $1.01(7)$ & -1.82 \\
\hline 316.691 & 100 & 28131.4 & $a^{5} P_{2}$ & 59698.6 & $z^{5} \mathrm{~F}_{2}^{\circ}$ & $1.89(7)$ & -1.55 & $2.09(7)$ & -1.50 \\
\hline 318.783 & 150 & 27801.4 & $a^{5} P_{3}$ & 59161.5 & $\mathrm{z}^{5} \mathrm{D}_{4}^{\circ}$ & 7.48(7) & -0.94 & $8.84(7)$ & -0.87 \\
\hline 320.725 & 100 & 25376.9 & $\mathrm{~b}^{3} \mathrm{~F}_{4}$ & 56547.3 & $\mathrm{z}^{5} \mathrm{~F}_{4}^{\circ}$ & $4.56(7)$ & -1.15 & $5.14(7)$ & -1.10 \\
\hline 323.332 & 100 & 27439.4 & $\mathrm{~b}^{3} \mathrm{~F}_{3}$ & 58358.5 & $\mathrm{z}^{5} \mathrm{~F}_{3}^{\circ}$ & $3.24(7)$ & -1.29 & $3.58(7)$ & -1.25 \\
\hline 323.895 & 20 & 28834.6 & $b^{3} F_{2}$ & 59698.6 & $\mathrm{z}^{5} \mathrm{~F}_{2}^{\circ}$ & $8.25(6)$ & -1.89 & $9.12(6)$ & -1.84 \\
\hline 324.049 & 5 & 32604.7 & $a^{3} G_{4}$ & 63454.9 & $\mathrm{z}^{3} \mathrm{~F}_{3}^{\circ}$ & $2.19(7)$ & -1.47 & $2.38(7)$ & -1.43 \\
\hline 326.427 & 20 & 29073.0 & $a^{5} P_{1}$ & 59698.6 & $\mathrm{z}^{5} \mathrm{~F}_{2}^{\mathrm{o}}$ & $7.02(6)$ & -1.95 & $7.76(6)$ & -1.91 \\
\hline 326.756 & 150 & 31730.5 & $a^{3} G_{5}$ & 62326.1 & $\mathrm{z}^{3} \mathrm{~F}_{4}^{\circ}$ & 7.07(7) & -0.94 & $8.25(7)$ & -0.88 \\
\hline $330.734^{*}$ & & 28131.4 & $a^{5} P_{2}$ & 58358.5 & $z^{5} \mathrm{~F}_{3}^{\circ}$ & $2.74(7)$ & -1.35 & $3.03(7)$ & -1.30 \\
\hline $336.361^{*}$ & & 32604.7 & $a^{3} G_{4}$ & 62326.1 & $\mathrm{z}^{3} \mathrm{~F}_{4}^{\circ}$ & $5.34(6)$ & -2.04 & $6.23(6)$ & -1.98 \\
\hline $339.547^{*}$ & & 31730.5 & $a^{3} G_{5}$ & 61173.1 & $\mathrm{z}^{5} \mathrm{G}_{4}^{\circ}$ & $1.46(7)$ & -1.60 & $1.55(7)$ & -1.57 \\
\hline $340.791^{*}$ & & 32604.7 & $a^{3} G_{4}$ & 61939.8 & $\mathrm{z}^{5} \mathrm{G}_{3}^{\circ}$ & $7.00(6)$ & -1.92 & 7.64(6) & -1.88 \\
\hline $343.451^{*}$ & & 27439.4 & $\mathrm{~b}^{3} \mathrm{~F}_{3}$ & 56547.3 & $\mathrm{z}^{5} \mathrm{~F}_{4}^{\circ}$ & $1.09(7)$ & -1.71 & $1.23(7)$ & -1.66 \\
\hline $345.266^{*}$ & & 36366.3 & $c^{3} \mathrm{P}_{2}$ & 65321.2 & $\mathrm{z}^{3} \mathrm{G}_{3}^{\circ}$ & $1.19(7)$ & -1.67 & $1.43(7)$ & -1.59 \\
\hline $347.776^{*}$ & & 27801.4 & $a^{5} P_{3}$ & 56547.3 & $\mathrm{z}^{5} \mathrm{~F}_{4}^{\circ}$ & $3.86(7)$ & -1.15 & $4.35(7)$ & -1.10 \\
\hline $352.232^{*}$ & & 36938.9 & $a^{3} D_{3}$ & 65321.2 & $\mathrm{z}^{3} \mathrm{G}_{3}^{\circ}$ & $1.01(7)$ & -1.73 & $1.21(7)$ & -1.65 \\
\hline $369.054^{*}$ & & 36366.3 & $c^{3} P_{2}$ & 63454.9 & $\mathrm{z}^{3} \mathrm{~F}_{3}^{\circ}$ & $5.34(6)$ & -1.96 & $5.80(6)$ & -1.93 \\
\hline $377.024^{*}$ & & 36938.9 & $a^{3} D_{3}$ & 63454.9 & $\mathrm{z}^{3} \mathrm{G}_{3}^{\circ}$ & $9.37(6)$ & -1.70 & $1.02(7)$ & -1.66 \\
\hline $391.561^{*}$ & & 36794.5 & $a^{3} \mathrm{H}_{5}$ & 62326.1 & $\mathrm{z}^{3} \mathrm{~F}_{4}^{\circ}$ & $5.05(6)$ & -1.93 & $5.89(6)$ & -1.87 \\
\hline $417.555^{*}$ & & 35787.2 & $a^{3} \mathrm{H}_{6}$ & 59729.4 & $\mathrm{z}^{5} \mathrm{G}_{5}^{\mathrm{o}}$ & $3.49(6)$ & -2.04 & $3.99(6)$ & -1.98 \\
\hline
\end{tabular}

Notes. ${ }^{(a)}$ From Sancho (1958). Wavelengths are given in vacuum and in air below and above $200 \mathrm{~nm}$, respectively. Starred values are deduced from experimental levels. ${ }^{(b)}$ This work : HFR+CPOL calculations (see the text). ${ }^{(c)}$ This work : HFR+CPOL values normalized by the laser measurements of the present work (see the text). 